Recently, however, R. Chakrabarty at the Desert Research Institute in Reno and the University of Nevada, M.A. Garro of the Desert Research Institute in Reno and Harvard University, J.G. Slowik of the University of Toronto, E.S. Cross of Boston College, T.B. Onasch of Aerodyne Research, Inc. in Billerica, Mass., and their colleagues have observed ensembles of cluster-dilute soot FAs with much lower $D_{\mathrm{f}}$ values, in the range of 1.2-1.5.

As reported in the June 12 issue of Physical Review Letters (DOI: 10.1103/PhysRevLett. 102.235504; \#235504), Chakrabarty and co-researchers used a pre-mixed etheneoxygen flame set-up that allowed them to vary the fuel-to-air equivalence ratio, $\phi$, from 2.3 to 5.0. After removing soot particles larger than $5 \mu \mathrm{m}$ from the sample with an impactor, the particles were bipolarly charged with a neutralizer, and then directed to two in-series electrostatic classifiers (ECs), which are widely used for particle sizing and operate with a combination of viscous and electrostatic force to select particles with a combination of charge and electrical mobility diameter, $D_{\mathrm{m}}$. The researchers said that this morphology segregation technique is based on the higher likelihood for more elongated particles becoming doubly charged than compact particles because less energy is required to add a second charge at a larger distance than at a short one. Constituting about $3 \%$ of the total submicron particle sample and following a Gaussian distribution with a peak $D_{\mathrm{m}}$ of about $460 \mathrm{~nm}$, elongated FAs were impacted onto clear polycarbonate filters for scanning electron microscopy (SEM) analysis.

Samples were coated with $1 \mathrm{~nm}$ Pt to prevent aerosol charging during SEM. The researchers imaged about 150 doublycharged FAs for each $\phi$ and corrected for the screening effect of $3 \mathrm{D}$ particles projected onto two dimensions. Image analysis showed a monotonic decrease in $D_{\mathrm{f}}$ from 1.51 to 1.20 as $\phi$ increases from 2.3 to 3.5 , which the researchers explained by suggesting that the electric field inside the flame causes a partial alignment of aggregates with a dipole moment and nonBrownian diffusion of charged monomers during the aggregation process. $D_{\mathrm{f}}$ should approach unity in the limit of vanishing Brownian motion.

The researchers said, "If the electrostatic force hypothesis can be verified, it may be possible to control the fractal dimension and associated properties of soot through application of a static electric field."

STEVEN TROHALAKI

\section{Surface-Modified Mesoporous Silica Nanoparticles Serve as Drug Carrier System}

The future of cancer therapies will draw from nanotechnology, cell biology, and organic chemistry. Drug delivery will play a vital role in realizing the potential success of newly discovered therapeutic agents, at least $40 \%$ of which are poorly soluble in water. Drug delivery matrices, targeting systems, and drug discovery can combine to create the ideal cancer therapy. By utilizing an interdisciplinary approach to address these requirements, J.M. Rosenholm of Åbo Akademi University, Finland, E. Peuhu of the University of Turku and Åbo Akademi University, Finland, and their colleagues have recently described their study using poly(ethylene imine) (PEI)-functionalized mesoporous silica nanoparticles as a cancer therapy delivery system.
As reported on July 2 in the online issue of Nano Letters (DOI: 10.1021/n1901589y), the research team created mesoporous silica nanoparticles with hyperbranched PEI conjugated to the surface. In order to design a cancer-targeting system, folic acid moieties, a ligand for a cell surface receptor that is over-expressed in cancer cells, were covalently attached to the PEI layer. By incubating nanoparticles with fluorescent dyes, delivery to cells could easily be monitored by confocal microscopy. Two different fluorescent dyes were used as models of poorly water-soluble compounds. Both molecules could be delivered into HeLa cells, a commonly studied cervical cancer cell line, without any leakage prior to endocytotic uptake. Furthermore, both fluorescent dyes could be co-delivered, suggesting the ability to administer multiple therapeutic agents simultaneously. As a final control, nanoparticles were incubated with both HeLa cells and a noncancerous cell line, epithelial HEK 293 (human embryonic kidney) cells. Minimal fluorescence was observed in HEK 293 cells, while HeLa cells were easily detected by fluorescence microscopy, demonstrating specificity of delivery between healthy and cancerous cell populations.

By combining aspects of materials chemistry, polymer chemistry, cell biology, and biotechnology, a very complex and innovative delivery matrix was designed. The researchers said that future research will be aimed at evaluating targetability, pharmacokinetics, toxicity, and particle degradation in animal tumor models in vivo.

DEVIN G. BARRETT

\section{Optical Transistor Developed with Single Molecule}

In order to reduce heat and increase the rate of data transfer in computers, scientists are searching for ways to produce integrated circuits that operate on the basis of photons instead of electrons. While optical transistor-like action has been previously reported in molecular systems involving many molecules as well as in single emitter configurations such as highfinesse micro-cavities or waveguides, these approaches are not generally suitable for photonic integrated circuits. Now V. Sandoghdar and colleagues at the Swiss Federal Institute of Technology have creat- ed an optical transistor using a single emitter configuration consisting of single molecules of dibenzanthanthrene doped in an $n$-tetradecane matrix.

As reported in the July 2 issue of Nature (DOI: 10.1038/nature08134; p. 76), the researchers discuss using one laser beam to prepare the quantum state of a single molecule of dibenzanthanthrene in a controlled fashion, such that they could significantly attenuate or amplify a second laser beam incident upon the molecule. This mode of operation is similar to that of a conventional transistor, in which electrical potential can be used to modulate a second signal. In order to enable this mode of operation, the researchers found that it was necessary to focus both laser beams to spot sizes approximately equal to the interaction cross-section of the single molecule. However, laser beams cannot generally be focused to spot sizes below the optical diffraction limit. On the other hand, at low temperatures, the cross-section for interaction of light with the dibenzanthanthrene molecule increases. The researchers found that by cooling the molecule down to $-272{ }^{\circ} \mathrm{C}$, they could achieve a regime in which the enlarged interaction cross-section of the molecule corresponded approximately to the diameters of the tightly focused laser 